\title{
Sleep quality and duration are associated with performance in maximal incremental test
}

\author{
B.M. Antunes ${ }^{\mathrm{a}, *}$, E.Z. Campos ${ }^{\mathrm{a}, \mathrm{b}}$, S.S. Parmezzani ${ }^{\mathrm{a}}$, R.V. Santos ${ }^{\mathrm{c}}$, E. Franchini ${ }^{\mathrm{d}}$, F.S. Lira ${ }^{\mathrm{a}, * *}$

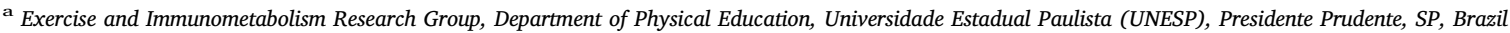 \\ b Universidade Federal de Pernambuco, Physical Education Department, Recife, Brazil \\ ${ }^{c}$ Department of Bioscience, Universidade Federal de São Paulo (UNIFESP), Santos, SP, Brazil \\ d Sport Department, School of Physical Education and Sport, University of São Paulo, São Paulo, Brazil
}

\section{A R T I C L E I N F O}

\section{Keywords:}

Sleep

Subjective sleep quality

Physical activity

Exercise

Anaerobic performance

\begin{abstract}
A B S T R A C T
Background and objective: Inadequate sleep patterns may be considered a trigger to development of several metabolic diseases. Additionally, sleep deprivation and poor sleep quality can negatively impact performance in exercise training. However, the impact of sleep duration and sleep quality on performance during incremental maximal test performed by healthy men is unclear. Therefore, the purpose of the study was to analyze the association between sleep pattern (duration and quality) and performance during maximal incremental test in healthy male individuals.

Methods: A total of 28 healthy males volunteered to take part in the study. Sleep quality, sleep duration and physical activity were subjectively assessed by questionnaires. Sleep pattern was classified by sleep duration ( $>7 \mathrm{~h}$ or $<7 \mathrm{~h}$ of sleep per night) and sleep quality according to the sum of measured points and/or scores by the Pittsburgh Sleep Quality Index (PSQI). Incremental exercise test was performed at 35 watts for untrained subjects, 70 watts for physically active subjects and 105 watts for well-trained subjects.

Results: $\mathrm{HR}_{\max }$ was correlated with sleep quality $(\mathrm{r}=0.411, \mathrm{p}=0.030)$ and sleep duration $(\mathrm{r}=-0.430$, $\mathrm{p}=0.022$ ). Participants reporting good sleep quality presented higher values of $\mathrm{W}_{\max }, \mathrm{VO}_{2 \max }$ and lower values of $\mathrm{HR}_{\max }$ when compared to participants with altered sleep. Regarding sleep duration, only $\mathrm{W}_{\max }$ was influenced by the amount of sleeping hours per night and this association remained significant even after adjustment by $\mathrm{VO}_{2 \max }$.

Conclusion: Sleep duration and quality are associated, at least in part, with performance during maximal incremental test among healthy men, with losses in $\mathrm{W}_{\max }$ and $\mathrm{HR}_{\max }$. In addition, our results suggest that the relationship between sleep patterns and performance, mainly in $\mathrm{W}_{\max }$, is independent of fitness condition.
\end{abstract}

\section{Introduction}

Poor sleep quality and sleep deprivation are associated with several metabolic dysfunctions. Inadequate sleep patterns may be considered a trigger to the development of diabetes mellitus [1], insulin resistance, hypertension and increased mortality [2]. Additionally, alteration in sleep patterns can influence other parameters associated with nutritional balance, such as food intake [3] body composition, and increased adiposity [4].

Regular exercise can modify several factors related to sleep patterns [5], improving sleep quality [6], duration and sleep latency [7], and promoting improvements upon falling asleep and staying asleep [8]. On the other hand, studies $[9,10]$ have shown that sleep deprivation and poor sleep quality can negatively affect performance during maximal incremental test and, consequently, impair exercise.

In a study conducted by Abedelmalek and colleagues [9], they observed that peak and mean power during 30-s Wingate test was affected by sleep deprivation in trained subjects. Moreover, Souissi and colleagues [10] verified that sleep deprivation at the end of the night decreases muscle strength and power when compared with sleep deprivation at the beginning of night among judoists.

In this context, studies have typically focused on the association between sleep quality and athletic performance or the relationship between sleep patterns and development of diseases and/or its progression [11-13]. However, few studies still seek to understand the impact of sleep patterns (duration and quality) on the parameters of perfor-

* Corresponding author at: Department of Physical Education, Universidade Estadual Paulista (UNESP), Roberto Simonsen Street, no 305 - Educational Center, $19060-900$ Presidente Prudente, Brazil.

*** Corresponding author.

E-mail addresses: ba.antunes2@gmail.com (B.M. Antunes), fabiolira@fct.unesp.br (F.S. Lira). 
Table 1

Median values and interquartile ranges of body composition and performance parameters in healthy men. Presidente Prudente/São Paulo, 2017.

\begin{tabular}{llll}
\hline & & Median & IQR (25th to 75th) \\
\hline \multirow{2}{*}{ Characteristics } & Age (years) & 29 & $24-33$ \\
& Body mass (kg) & 75.5 & $70.6-78.9$ \\
& Height $(\mathrm{cm})$ & 178 & $171-182$ \\
& BMI $\left(\mathrm{kg} / \mathrm{m}^{2}\right)$ & 23.9 & $22.9-24.4$ \\
& $\mathrm{BF}(\%)$ & 16.2 & $13.6-20.5$ \\
& $\mathrm{FM}(\mathrm{kg})$ & 11.9 & $9.8-15.3$ \\
& $\mathrm{LM}(\mathrm{kg})$ & 62.0 & $58.1-66.1$ \\
& $\mathrm{~W}_{\text {Max }}(\mathrm{watts})$ & 202 & $172-270$ \\
& $\mathrm{HR}_{\text {res }}(\mathrm{bpm})$ & 72 & $66-79$ \\
& $\mathrm{HR}_{\max }(\mathrm{bpm})$ & 189 & $184-195$ \\
& $\mathrm{VO}_{2 \max }\left(\mathrm{mL} \cdot \mathrm{kg}^{-1} \cdot \mathrm{min}^{-1}\right)$ & 53 & $44-59$ \\
\hline
\end{tabular}

$\mathrm{IQR}=$ interquartile range; $\mathrm{BMI}=$ body mass index; $\mathrm{BF}=$ body fat; $\mathrm{FM}=$ fat mass; $\mathrm{LM}=$ lean mass; $\mathrm{W}_{\max }=$ maximum power output; $\mathrm{HR}_{\mathrm{res}}=$ resting heart rate; $\mathrm{HR}_{\max }=$ maximal heart rate; $\mathrm{VO}_{2 \max }=$ maximal oxygen uptake.

mance during different incremental protocols among healthy subjects, as well as how fitness condition may influence sleep pattern [14-16]. Therefore, the aim of the present investigation was to test the following hypothesis: (i) performance during maximal incremental test is associated with sleep patterns, as well as sleep quality and duration, and (ii) these alterations may be fitness-independent.

\section{Methods}

\subsection{Participants and procedures}

A sample of 28 healthy male volunteers was recruited to participate in this study and the characteristics of the subjects are presented in Table 1. The inclusion criteria for the study were: I) Body Mass Index $(\mathrm{BMI}) \leq 25 \mathrm{~kg} / \mathrm{m}^{2}$, II) no health problems preventing high-intensity efforts, and III) no smoking, no alcohol intake or illicit drugs. All volunteers were informed about the study procedures and signed a consent form. All procedures were approved by the University Ethics Committee (CAAE: 31168714.6.0000.5402).

\subsection{Experimental protocol}

All tests were performed in the same laboratory, by specialized professionals, between 8:00 am and 12:00 pm. Participants visited the laboratory once for anthropometric and body composition measurements, as well as to complete the questionnaires about sleep quality (short version of Pittsburgh Sleep Quality Index - PSQI) and physical activity (International Physical Activity Questionnaire - IPAQ).

All participants performed the graded exercise test to exhaustion. They were instructed not to consume alcohol, caffeine and/or stimulants of any kind during the $24 \mathrm{~h}$ prior to the test. Additionally, they were also instructed not to eat a meal less than two hours before the test. The tests were performed under controlled conditions (mean temperature $=22.1{ }^{\circ} \mathrm{C}$; mean relative humidity: $55 \%$; mean barometric pressure: $731.3 \mathrm{mmHg}$ ).

\subsection{Maximal incremental test}

Participants took part in a maximal incremental test in cycle ergometer (Inbrasport CG-04, Embramed, Porto Alegre, Brasil) consisting of 3-min stages until voluntary exhaustion to determine performance and physical fitness parameters, by measurements of maximum power output, oxygen uptake, resting and maximal heart rate, respectively.

The initial workload was defined according to the reported fitness condition assessed via IPAQ and subsequently confirmed by maximal oxygen uptake, as follows: $35 \mathrm{~W}$ for the untrained subjects, $70 \mathrm{~W}$ for physically active subjects, and $105 \mathrm{~W}$ for the well-trained subjects. In all cases, a $25 \mathrm{~W}$ increment at each stage, and cycling frequency between 70 and $90 \mathrm{rpm}$ was used $[17,18]$. The oxygen uptake $\left(\mathrm{VO}_{2}\right)$ was assessed by a breath-by-breath respiratory gas analyzer (Quark PFT, Cosmed ${ }^{\circledR}$, Rome, Italy). The variables measured during the test were: maximal oxygen uptake $\left(\mathrm{VO}_{2 \max }\right)$, assumed as the highest 30 -s mean observed during the incremental test; maximal heart rate $\left(\mathrm{HR}_{\max }\right)$ (Polar S810i, Polar Electro Oy ${ }^{\circledR}$, Kempele, Finland); and maximum power output $\left(\mathrm{W}_{\max }\right)$. The exhaustion criteria were confirmed by the following variables: gas exchange ratio $>1.1, \mathrm{HR}_{\max }>90 \%$ of the maximum expected for the age and rating of perceived exertion $(\mathrm{RPE})>18$. All performance variables were classified and divided in groups according to the 50th and 75th percentiles.

\subsection{Body composition}

Body mass (BM) was measured with an electronic scale (precision $0.1 \mathrm{~kg}$ [Filizzola PL 150, Filizzola ${ }^{\circledR}$ Ltda]) and height with a wallmounted stadiometer (precision $0.1 \mathrm{~cm}$ [Sanny ${ }^{\circledR}$, São Paulo, Brazil]), with the subjects wearing light clothing and no shoes. BMI was calculated through weight and height measurements. To estimate body density we used the equation developed by Jackson and Pollock [19], which considered seven skinfold measurements. Skinfold caliper [precision $1 \mathrm{~mm}$ (Lange Beta Technology Incorpored, Cambridge, Maryland)] was used to measure skinfold thickness. To calculate body fat percentage, we used the Siri equation [20]. All anthropometric measurements were performed by a trained researcher, using standardized techniques.

\subsection{Self-reported sleep quality}

Sleep quality was assessed by personal interview using the short version of the Pittsburgh Sleep Quality Index (PSQI), which was validated for the Brazilian population by Falavigna [21]. The questionnaire obtains information about sleep difficulty, overnight sleep disruption, perception of sleep quality and daily amount of hours dedicated to sleep per night [22-24].

Participants were asked to answer the 10 questions of the self-rated questionnaire to determine sleep quality during the week prior to the incremental test. Sleep duration was divided into two categories: $>7 \mathrm{~h}$ of sleep per night (scale 0 ) and $<7 \mathrm{~h}$ (scale 1); additionality, sleep quality was classified according to the sum of points $(<24$ points $=$ good sleep quality; $>25$ points = altered sleep quality); and using scores $(0=$ highly altered sleep; $1=$ moderately altered sleep; 2 = slightly altered sleep; $3=$ good sleep).

\subsection{Data analysis}

Kolmogorov-Smirnov test analyzed normality of data. Descriptive statistics were expressed as median and interquartile range (IQR). Correlations between performance and sleep quality were calculated using Spearman correlation, and associations were identified by ChiSquare test. For Chi-square association analyses, the sample was divided into groups according to sleep quality (good sleep $\leq 24$ points or sleep disorder $\geq 24$ points in PSQI), sleep duration ( $>$ or $<7 \mathrm{~h}$ ), and performance during maximal incremental test according to percentiles ( $>$ p50 or $<$ p50; $>$ p75 or $<$ p75). As subjects with different training status present different power-oxygen consumption relationship (i.e. more aerobic trained individuals have lower oxygen uptake for given absolute exercise intensity) we tested the association between $\mathrm{W}_{\max }$ and sleep patterns controlling by $\mathrm{VO}_{2 \text { max. }}$. For this, the Chi-Square association analyses were carried out according to maximal oxygen uptake percentiles ( $>$ p75 and $<$ p75). All statistical analyses were performed in the software SPSS (release 12.0) and statistical significance (p-value) was set at 0.05 . 

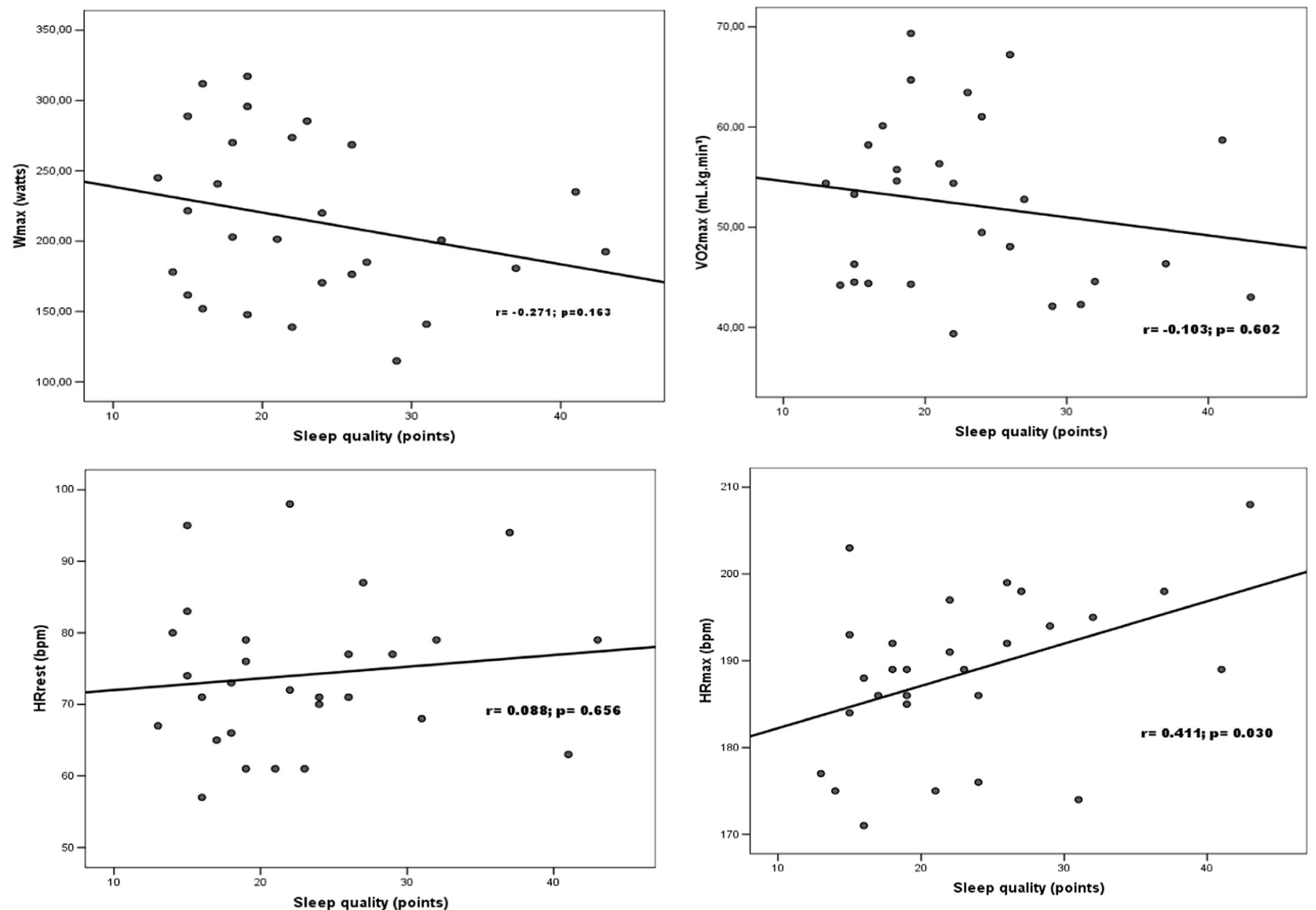

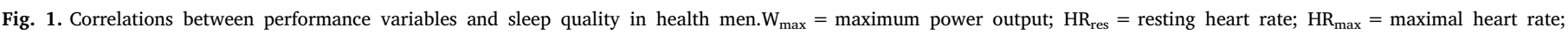
$\mathrm{VO}_{2 \max }=$ maximal oxygen uptake.

\section{Results}

Descriptive results are shown in Table 1 according to percentiles of performance and body composition parameters obtained by maximal incremental test.

Fig. 1 shows the relationship between sleep quality and performance parameters obtained during the incremental test. Positive correlation was observed only between maximal heart rate and sleep quality.

Table 2 shows the association between sleep quality and performance parameters. Individuals with good sleep quality presented better performance during the incremental test with higher values of $\mathrm{W}_{\max }$ and $\mathrm{VO}_{2 \max }$ and lower values of $\mathrm{HR}_{\text {max }}$. Furthermore, $58.3 \%$ of the individuals with sleep disorders were above the average for hemodynamic variables when submitted to the incremental test.

Correlation analyses between sleep duration and sleep quality scores (good sleep $\leq 24$ points or sleep disorder $\geq 24$ points) are presented in Fig. 2. The results suggest that individuals with higher sleep hours had better sleep quality.

Establishing the cutoff point in seven hours, we observed that $57.1 \%$ of the sample slept less than seven hours per night and achieved lower $\mathrm{W}_{\max }$ during maximal incremental test (Table 3 ). On the other hand, $85.7 \%$ of the individuals reporting more than seven hours of sleep presented better performance, reaching higher $\mathrm{W}_{\text {max }}$.

Significant association between sleep duration and $\mathrm{W}_{\max }$ remained significant even after adjustment, showing that $58.8 \%$ of the participants who slept less than seven hours reached maximum power below the 75th percentile, and the maximum consumption of oxygen below
Table 2

Associations between sleep quality and performance parameters in the maximal incremental test. Presidente Prudente/São Paulo, 2017.

\begin{tabular}{|c|c|c|c|c|c|c|}
\hline & \multicolumn{2}{|c|}{ Good sleep } & \multicolumn{2}{|c|}{ Altered sleep } & \multicolumn{2}{|c|}{ Chi-square test } \\
\hline & $\mathrm{n}$ & $\%$ & $\mathrm{n}$ & $\%$ & $\chi^{2}$ & $\mathrm{p}$ \\
\hline \multicolumn{7}{|c|}{$\mathrm{W}_{\max }$ (watts) } \\
\hline$<$ p50 & 7 & 50.0 & 7 & 50.0 & 4.094 & $0.043^{*}$ \\
\hline$>$ p50 & 12 & 85.7 & 2 & 14.3 & & \\
\hline \multicolumn{7}{|c|}{$\mathrm{HR}_{\text {res }}(\mathrm{bpm})$} \\
\hline$<\mathrm{p} 50$ & 11 & 78.6 & 3 & 21.4 & 1.474 & 0.225 \\
\hline$>$ p50 & 8 & 57.1 & 6 & 42.9 & & \\
\hline \multicolumn{7}{|c|}{$\mathrm{HR}_{\max }(\mathrm{bpm})$} \\
\hline$<\mathrm{p} 50$ & 14 & 87.5 & 2 & 12.5 & 6.604 & $0.010^{*}$ \\
\hline$>\mathrm{p} 50$ & 5 & 41.7 & 7 & 58.3 & & \\
\hline \multicolumn{7}{|c|}{$\mathrm{VO}_{2 \max }\left(\mathrm{mL} \cdot \mathrm{kg} \cdot \mathrm{min}^{-1}\right)$} \\
\hline$<$ p50 & 7 & 50.0 & 7 & 50.0 & 4.094 & $0.043^{*}$ \\
\hline$>\mathrm{p} 50$ & 12 & 85.7 & 2 & 14.3 & & \\
\hline
\end{tabular}

$\mathrm{W}_{\max }=$ maximum power output; $\mathrm{HR}_{\mathrm{res}}=$ resting heart rate; $\mathrm{HR}_{\max }=$ maximal heart rate; $\mathrm{VO}_{2 \max }=$ maximal oxygen uptake.

* Chi-square $<0.05$.

the 75th percentile. On the other hand, $100 \%$ of the participants who slept more than seven hours reached higher maximal aerobic power during the incremental test $(>\mathrm{p} 75)\left(\chi^{2}=4.492 ; \mathrm{p}=0.034\right)$.

\section{Discussion}

The aim of the present study was to evaluate the influence of sleep 


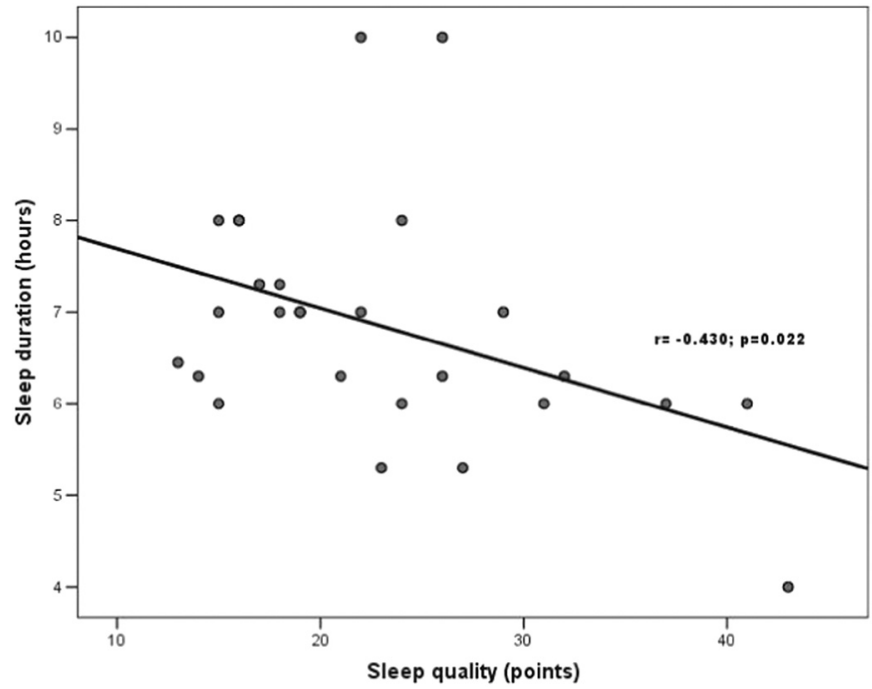

Fig. 2. Relationship between sleep duration and sleep quality.

Table 3

Associations between sleep duration and percentiles of performance parameters during the maximal incremental test. Presidente Prudente/São Paulo, 2017.

\begin{tabular}{|c|c|c|c|c|c|c|}
\hline & \multicolumn{2}{|c|}{$<7 \mathrm{~h}$} & \multicolumn{2}{|c|}{$>7 \mathrm{~h}$} & \multicolumn{2}{|c|}{ Chi-square } \\
\hline & $\mathrm{n}$ & $\%$ & $\mathrm{n}$ & $\%$ & $\chi^{2}$ & $\mathrm{p}$ \\
\hline \multicolumn{7}{|c|}{$\mathrm{W}_{\max }$ (watts) } \\
\hline$<$ p50 & 8 & 57.1 & 6 & 42.9 & 1.292 & 0.256 \\
\hline$<$ p75 & 12 & 57.1 & 9 & 42.9 & 3.877 & 0.049 \\
\hline \multicolumn{7}{|c|}{$\mathrm{HR}_{\mathrm{res}}(\mathrm{bpm})$} \\
\hline$<$ p50 & 6 & 42.9 & 8 & 57.1 & 0.144 & 0.705 \\
\hline$<$ p75 & 9 & 40.9 & 13 & 59.1 & 1.257 & 0.262 \\
\hline \multicolumn{7}{|c|}{$\mathrm{HR}_{\max }(\mathrm{bpm})$} \\
\hline$<$ p50 & 7 & 43.8 & 9 & 56.3 & 0.108 & 0.743 \\
\hline$<$ p75 & 8 & 38.1 & 13 & 61.9 & 2.345 & 0.126 \\
\hline \multicolumn{7}{|c|}{$\mathrm{VO}_{2 \max }\left(\mathrm{mL} \cdot \mathrm{kg}^{-1} \cdot \min ^{-1}\right)$} \\
\hline$<$ p50 & 8 & 57.1 & 6 & 42.9 & 1.292 & 0.256 \\
\hline$<$ p75 & 10 & 47.6 & 11 & 52.4 & 0.048 & 0.827 \\
\hline
\end{tabular}

$\mathrm{W}_{\max }=$ maximum power output; $\mathrm{HR}_{\mathrm{res}}=$ resting heart rate; $\mathrm{HR}_{\max }=$ maximal heart rate; $\mathrm{VO}_{2 \max }=$ maximal oxygen uptake.

* Chi-square $<0.05$

patterns on the performance during incremental test and, additionally, to investigate if sleep quality alterations is fitness-dependent. According to our initial hypothesis, participants with greater physical fitness assessed by performance in the incremental maximal test - presented better sleep quality than participants with lower physical fitness. Furthermore, our results showed that, after adjustment, $\mathrm{W}_{\max }$ was the main performance parameter affected by sleep patterns and appear to be independent of $\mathrm{VO}_{2 \max }$ (i.e., it seems to be fitness-independent.) These findings elucidate the importance of adopting and maintaining a healthy lifestyle, independently of the physical fitness level, evidencing that not only nutritional and daily habits, but also the nocturnal habits are determinant factors to have a good performance in the practice of physical exercise as well as to benefit from the positive aspects associated with exercise training.

It is important to highlight in our findings that sleep patterns had impact mainly in measures of $\mathrm{W}_{\max }, \mathrm{VO}_{2 \max }$ and $\mathrm{HR}_{\max }$, revealing the harmful outcomes of poor sleep quality in exercise among healthy men. Therefore, sleep patterns seems to be an important variable that affects regular exercise, and is determinant to achieve good performance in exercise. Previous studies have shown associations between performance impairment in incremental test and sleep quality $[9,10]$.

In a study conducted by Souissi and colleagues [16], aimed to analyze the effects of four hours of sleep deprivation (beginning or end of the night) on anaerobic performance using Wingate test, it was observed that peak power and mean power were affected by sleep deprivation mainly by reduction of sleep time in the end of the night. Although we did not evaluate sleep deprivation, our results are in accordance with the scientific literature, showing impairments and/or low indices of power in subjects who sleep less than seven hours per night and have poor sleep quality.

In this context, the maximal oxygen uptake is also affected and altered by sleep quality in healthy men. Alterations in this cardiopulmonary parameter have also been observed in athletes, observing relationship between training conditions, sleep duration and sleep efficiency. Additionally, in the group characterized as "functionally overreached", there was a higher prevalence of upper respiratory tract infections, suggesting that sleep quality and incidence of illness are symptoms of short-term "overreaching" [14].

Lang and colleagues [15] investigated the relationship between physical activity and sleep in 56 adolescents using subjective and objective measurements. The authors verified that adolescents who reported high physical activity levels had shorter sleep onset latencies and longer REM latency. In addition, the longer REM latency was also associated with physical activity by objective measurements (electroencephalogram assessments (EEG)). In a different perspective, these results support in parts our findings, suggesting that fitness condition and physical activity level can influence sleep pattern, causing a mutual interaction between the variables. It is important to highlight that after adjustment of performance parameters, by maximal oxygen uptake, the maximal power remained associated with sleep duration and, consequently, sleep quality. Therefore, our results suggest that the association between performance and sleep quality is fitness conditionindependent in healthy men.

The regular practice of exercise is capable to promote benefits by providing significant alteration in body composition, metabolism regulation, and control of autonomic nervous system. These outcomes occur due to the increase of vagal modulation of the heart caused by greater heart rate variability $[25,26]$, which are recommended for adequate sleep [27]. However, the alterations mediated by exercise are dependent of several daily habits, such as duration and sleep quality, given that sleep is a crucial recovery period.

In a study conducted by Myllymäki and colleagues [28], they investigated the effects of exercise intensity and duration, performed at 6 p.m., on nocturnal heart rate behavior and sleep quality. It was observed that the increase in intensity and/or duration caused delayed recovery of nocturnal cardiac autonomic modulation. Moreover, there was a relationship between duration and changes in nocturnal heart rate variability. Regarding sleep quality, the authors suggested that independently of intensity and duration, aerobic exercise is not able to result in disturbance of sleep pattern.

Recent study performed by Castro-Diehl and colleagues [29] verified the associations between shorter sleep duration (less than six hours per night), poor sleep quality and markers of autonomic nervous system, as heart rate (HR) and high-frequency HR variability (HF-HRV). The authors observed that subjects who slept less than six hours had higher heart rate and had lower baseline HF-HRV when compared to subjects that slept seven hour or more, proving the relationship between short sleep duration and autonomic markers.

The findings from Myllymäki's and Castro-Diehl's [28,29] studies may help us to understand our results and to identify a significant relationship between sleep quality and $\mathrm{HR}_{\max }$. These studies lead us to understand that changes in sleep patterns cause a significant loss in the autonomic response, which affects the behavior of these variables in stress condition. It is important to highlight that some variables may influence the results of hemodynamic parameters, such as age. Our results of $\mathrm{HR}_{\max }$ remained associated with sleep quality after adjustment by age, but only among men $\geq 30$ years old. In this group ( $\geq 30$ years old), $100 \%$ of the subjects who had good sleep showed lower $\mathrm{HR}_{\max }$ on the incremental test. On the other hand, $75 \%$ of the 
individuals that had altered sleep showed higher $\mathrm{HR}_{\max }$ (data not shown).

Despite the significant results presented in this study, some limitations were identified and should be considered when interpreting the results. The main limitation is the absence of sleep quality evaluation by objective measurements. Data obtained through subjective evaluations, such as questionnaires, are relevant, have good applicability, reproducibility and are inexpensive; however, information coming from direct measurements is important to confirm the relationship between the parameters analyzed. In addition, other general limitations may be highlighted such as restricted and specific population, making necessary studies with other groups (women, young, sedentary, senior athletes), as well as the reproducibility of the results in other protocols, such as in tests performed on a treadmill.

Our findings showed that sleep quality is associated with performance during maximum incremental test among healthy men; however, future studies should be conducted in this population in order to investigate the influence of performance on sleep quality. Therefore, we conclude that duration and sleep quality (general or specific alterations in sleep pattern) are, at least in part, associated with performance during maximal incremental test among healthy men, especially with lower maximal aerobic power and maximal heart rate. In addition, our results suggested that this relationship with performance, mainly on power, is independent on fitness condition.

\section{Conflict of interest}

The authors have no conflicts of interest that are directly relevant to the content of this article.

\section{Acknowledgments}

Barbara de Moura Antunes thanks São Paulo Research Foundation (FAPESP) for their support (no 2014/08003-1); Fabio Santos Lira thanks National Counsel of Technological and Scientific Development (CNPq) for their support (305263/2015-3) and Eduardo Zapaterra Campos thanks National Counsel of Technological and Scientific Development (CNPq) for their support (no 401676/2014-15).

\section{References}

[1] F.P. Cappuccio, L. D'Elia, P. Strazzullo, M.A. Miller, Quantity and quality of sleep and incidence of type 2 diabetes, Diabetes Care 33 (2) (2010 Feb) 414-420, http:// dx.doi.org/10.2337/dc09-1124.

[2] M.A. Grandner, L. Hale, M. Moore, N.P. Patel, Mortality associated with short sleep duration: the evidence, the possible mechanisms, and the future, Sleep Med. Rev. 14 (3) (2010 Jun) 191-203, http://dx.doi.org/10.1016/j.smrv.2009.07.006.

[3] C.A. Crispim, I.Z. Zimberg, B.G. dos Reis, R.M. Diniz, S. Tufik, M.T. de Mello, Relationship between food intake and sleep pattern in healthy individuals, J. Clin. Sleep Med. 7 (6) (2011 Dec 15) 659-664, http://dx.doi.org/10.5664/jcsm.1476.

[4] M.F. Hjorth, A. Sjödin, S.M. Dalskov, C.T. Damsgaard, K.F. Michaelsen, A. BiltoftJensen, R. Andersen, C. Ritz, J.P. Chaput, A. Astrup, Sleep duration modifies effects of free ad libitum school meals on adiposity and blood pressure, Appl. Physiol. Nutr. Metab. 41 (1) (2016 Jan) 33-40, http://dx.doi.org/10.1139/apnm-2015-0319.

[5] A. Maculano Esteves, C. Ackel-D'Elia, S. Tufik, M.T. De Mello, Sleep patterns and acute physical exercise: the effects of gender, sleep disturbances, type and time of physical exercise, J. Sports Med. Phys. Fitness 54 (6) (2014 Dec) 809-815.

[6] K. Mansikkamäki, J. Raitanen, C.H. Nygård, R. Heinonen, T. Mikkola, EijaTomás, R. Luoto, Sleep quality and aerobic training among menopausal women- a randomized controlled trial, Maturitas 72 (4) (2012 Aug) 339-345, http://dx.doi. org/10.1016/j.maturitas.2012.05.003.

[7] F. Sharif, M. Seddigh, I. Jahanbin, S. Keshavarzi, The Effect of aerobic exercise on quantity and quality of sleep among elderly people referring to health centers of Lar City, southern of Iran; a randomized controlled clinical trial, Curr. Aging Sci. 8 (3) (2015) 248-255.

[8] J.R. Alley, J.W. Mazzochi, C.J. Smith, D.M. Morris, S.R. Collier, Effects of resistance exercise timing on sleep architecture and nocturnal blood pressure, J. Strength Cond. Res. 29 (5) (2015 May) 1378-1385, http://dx.doi.org/10.1519/JSC. 0000000000000750.

[9] S. Abedelmalek, H. Chtourou, A. Aloui, C. Aouichaoui, N. Souissi, Z. Tabka, Effect of time of day and partial sleep deprivation on plasma concentrations of IL-6 during a short-term maximal performance, Eur. J. Appl. Physiol. 113 (1) (2013 Jan) 241-248, http://dx.doi.org/10.1007/s00421-012-2432-7.

[10] N. Souissi, H. Chtourou, A. Aloui, O. Hammouda, M. Dogui, A. Chaouachi, K. Chamari, Effects of time-of-day and partial sleep deprivation on short-term maximal performances of judo competitors, J. Strength Cond. Res. 27 (9) (2013 Sep) 2473-2480, http://dx.doi.org/10.1519/JSC.0b013e31827f4792.

[11] L. Isacco, J. Roche, S. Quinart, D. Thivel, V. Gillet, V. Nègre, F. Mougin, Cardiometabolic risk is associated with the severity of sleep-disordered breathing in children with obesity, Physiol. Behav. 170 (2017 Mar 1) 62-67, http://dx.doi.org/ 10.1016/j.physbeh.2016.12.018.

[12] J.C. Chen, M.A. Espeland, R.L. Brunner, L.C. Lovato, R.B. Wallace, X. Leng, L.S. Phillips, J.G. Robinson, J.M. Kotchen, K.C. Johnson, J.E. Manson, M.L. Stefanick, G.E. Sarto, W.J. Mysiw, Sleep duration, cognitive decline, and dementia risk in older women, Alzheimers Dement. 12 (1) (2016 Jan) 21-33, http://dx.doi.org/10.1016/j.jalz.2015.03.004.

[13] M.N. Rao, T.C. Neylan, C. Grunfeld, K. Mulligan, M. Schambelan, J.M. Schwarz, Subchronic sleep restriction causes tissue-specific insulin resistance, J. Clin. Endocrinol. Metab. 100 (4) (2015 Apr) 1664-1671, http://dx.doi.org/10.1210/jc. 2014-3911.

[14] C. Hausswirth, J. Louis, A. Aubry, G. Bonnet, R. Duffield, Y.L.E. Meur, Evidence of disturbed sleep and increased illness in overreached endurance athletes, Med. Sci. Sports Exerc. 46 (5) (2014) 1036-1045, http://dx.doi.org/10.1249/MSS. 0000000000000177.

[15] C. Lang, S. Brand, A.K. Feldmeth, E. Holsboer-Trachsler, U. Pühse, M. Gerber, Increased self-reported and objectively assessed physical activity predict sleep quality among adolescents, Physiol. Behav. 120 (2013 Aug 15) 46-53, http://dx. doi.org/10.1016/j.physbeh.2013.07.001.

[16] N. Souissi, M. Souissi, H. Souissi, K. Chamari, Z. Tabka, M. Dogui, D. Davenne, Effect of time of day and partial sleep deprivation on short-term, high-power output, Chronobiol. Int. 25 (6) (2008) 1062-1076, http://dx.doi.org/10.1080/ 07420520802551568

[17] R.A.C. Caritá, F. Caputo, C.C. Greco, B.S. Denadai, Aerobic fitness and amplitude of the exercise intensity domains during cycling, Rev. Bras. Med. Esporte 19 (4) (2013), http://dx.doi.org/10.1590/S1517-86922013000400009.

[18] F. Caputo, B.S. Denadai, The highest intensity and the shortest duration permitting attainment of maximal oxygen uptake during cycling: effects of different methods and aerobic fitness level, Eur. J. Appl. Physiol. 103 (1) (2008 May) 47-57, http:// dx.doi.org/10.1007/s00421-008-0670-5.

[19] A.S. Jackson, M.L. Pollock, Generalized equations for predicting body density of men, Br. J. Nutr. 40 (3) (1978 Nov) 497-504.

[20] W.E. Siri, Body composition from fluid spaces and density: analysis of methods 1961, Nutrition 9 (5) (1993 Sep-Oct) 480-491 (discussion 480, 492).

[21] A. Falavigna, M.L. de Souza Bezerra, A.R. Teles, F.D. Kleber, M.C. Velho, R.C. da Silva, T. Mazzochin, J.T. Santin, G. Mosena, G.L. de Braga, F.L. Petry, M.F. de Lessa Medina, Consistency and reliability of the Brazilian Portuguese version of the MiniSleep Questionnaire in undergraduate students, Sleep Breath. 15 (3) (2011 Sep) 351-355, http://dx.doi.org/10.1007/s11325-010-0392-x.

[22] L. Gallicchio, B. Kalesan, Sleep duration and mortality: a systematic review and meta-analysis, Res. 18 (2) (2009 Jun) 148-158, http://dx.doi.org/10.1111/j.1365 2869.2008.00732.x.

[23] M.A. Grandner, N.P. Patel, From sleep duration to mortality: implications of metaanalysis and future directions, J. Sleep Res. 18 (2) (2009 Jun) 145-147, http://dx. doi.org/10.1111/j.1365-2869.2009.00753.x.

[24] M.A. Grandner, L. Hale, M. Moore, N.P. Patel, Mortality associated with short sleep duration: the evidence, the possible mechanisms, and the future, Sleep Med. Rev. 14 (3) (2010 Jun) 191-203, http://dx.doi.org/10.1016/j.smrv.2009.07.006.

[25] J.F. Thayer, S.S. Yamamoto, J.F. Brosschot, The relationship of autonomic imbalance, heart rate variability and cardiovascular disease risk factors, Int. J. Cardiol. 141 (2) (2010 May 28) 122-131, http://dx.doi.org/10.1016/j.ijcard.2009. 09.543.

[26] A.J. Hautala, A.M. Kiviniemi, M.P. Tulppo, Individual responses to aerobic exercise: the role of the autonomic nervous system, Neurosci. Biobehav. Rev. 33 (2) (2009 Feb) 107-115, http://dx.doi.org/10.1016/j.neubiorev.2008.04.009.

[27] American Academy of Sleep Medicine, International Classification of Sleep Disorders, Revised: Diagnostic and Coding Manual, American Academy of Sleep Medicine, Chicago, 2001.

[28] T. Myllymäki, H. Rusko, H. Syväoja, T. Juuti, M.L. Kinnunen, H. Kyröläinen, Effects of exercise intensity and duration on nocturnal heart rate variability and sleep quality, Eur. J. Appl. Physiol. 112 (3) (2012 Mar) 801-809, http://dx.doi.org/10. 1007/s00421-011-2034-9.

[29] C. Castro-Diehl, A.V. Diez Roux, S. Redline, T. Seeman, P. McKinley, R. Sloan, S. Shea, Sleep duration and quality in relation to autonomic nervous system measures: the multi-ethnic study of atherosclerosis (MESA), Sleep 39 (11) (2016 Nov 1) 1927-1940. 